as the responsibilities. Certainly it should help to dispel whatever there may have been in the atmosphere of the Lagos Conference which hinders a constructive approach to the immense tasks confronting the emergent countries of Africa, or the full co-operation of scientists everywhere in assisting those countries to discharge these tasks successfully.

\section{MATHEMATICAL METHODS IN U.S.S.R. TECHNOLOGY}

\section{A Course of Higher Mathematics}

By V. I. Smirnov. Translated by D. E. Brown. Translation edited by I. N. Sneddon. Vol. 3, Part 1: Linear Algebra. Pp. ix +324. 63s. net. Vol. 3, Part 2: Complex Variables/Special Functions. Pp. $\mathrm{x}+702$. 110s. net. Vol. 4: Integral Equations and Partial Differential Equations. Pp. xiii +814 . 126s. net. Vol. 5: Integration and Functional Analysis. Pp. xiv +638 . 126s. net. (International Series of Monographs in Pure and Applied Mathematics, Vols. 60, 61 and 62.) (London and New York: Pergamon Press, 1964.)

$\mathrm{T}$ HESE volumes complete the translation of Smirnov's massive treatise, which, it must be remembered, was designed with the needs of physicists and engineers in mind. There is a hint in the author's preface to Volume 5 that a further volume on differential operators might be written some day.

One noteworthy fact is that modern algebraic theories are regarded as being as important as calculus and the theory of functions. Volume 3, Part 1, on linear algebra, is a self-contained text on determinants, linear transforma. tions, matrices, quadratic forms and group theory. While the range is practical enough to include numerical illustrations of the reduction of quadratic forms to canonical form, it is abstract enough to deal with the elements of function spaces and Hilbert space, a pointer to the thorough and recondite work on functional analysis in Volume 5 .

The classical theory of functions of a complex variable occupies Part 2 of Volume 3. Much of it will serve the young mathematician as well as the technologist, but the emphasis is, very properly, on elaborating the powerful techniques of this theory. Thus Cauchy's fundamental theorem on the integral of a regular function $f(z)$ is proved on the assumption that $f$ has a continuous derivative, so that Green's lemma can be used; the possibility of removing the continuity condition is mentioned, but not pursued. The simple mapping theorem is proved, but Riemann's existence theorem for the mapping of a connected domain on a circle is quoted without proof. This, of course, does not hinder a full development of the technique of conformal mapping, including the SchwarzChristoffel transformation, and sections on applications to electricity and to hydrodynamics (where we have to recognize Blasius's theorem under Zhukovskij's name). The formalities of the residue calculus, and the theory of special functions (Legendre, Bessel, the elliptic functions) balance elegant formalism neatly against practical applications. One unusual feature is a chapter on functions of several complex variables, while one sad one is the use of $Q_{n}$ for the Laguerre polynomials.

The main theme of Volume 4 is that of boundary value problems in mathematical physies. Two hundred pages on integral equations of Fredholm and Volterra type, with singular equations of the Cauchy and Fourier character, and 100 pages on the calculus of variations, with the nature of extrema, strong and weak variations, and an excursus which yields the whole of the fundamental theory of linearized elasticity on a variational basis, form a solid foundation for the study of boundary value problems. In a long chapter on the theory of partial differential equations, characteristics are made the core of the work, and the culminating chapter deals with boundary value problems in ordinary differential equations, with the use of Green's function, and with elliptic, parabolic and hyperbolic partial differential equations, in great detail and thoroughness. Throughout this section, we are constantly brought back to physical problems in electro-magnetism, heat, elasticity. Nothing much is said about approximative and numerical methods; other well-known Russian texts deal fully with these aspects.

Up to this point, the content of this work resembles, for example, that of Sir Harold and Lady Jeffreys' Methods of Mathematical Physics, though the Russian book is much more voluminous. But in the final volume, the abstract disciplines may startle the theoretical physicist, and, a fortiori, the engineer. Set theory, the Stieltjes and the Lebesgue-Stieltjes integrals, the theory of completely additive set functions belong to quite advanced mathematics; and when this is followed by a detailed study of metric and normed spaces, and of Hilbert space, some readors are going to ask what all this is in aid of. But the general theory of operators, with the theory of functionals as a special case, can scarcely be understood without the concept of an operator as a transformation between elements in two metric or normed abstract spaces. The author obviously owes much to Sobolev's work on the applications of functional analysis to mathematical physics, but even so, this volume is the least satisfactory of the series when it comes to relating the mathematical content to physical problems. Much has to be taken for granted.

Prof. Sneddon, in his introductions to each volume, rightly indicates the high regard in which this major work is held by applied mathematicians of all kinds in the U.S.S.R. In Britain, the engineer and the technologist have, on the whole, been content to rely on the ingenuity and industry whereby they can extract the maximum amount of value out of a comparatively elementary knowledge of mathematics. How long will this suffice? Recently, a panel of mathematicians in the United States made a survey of Soviet contributions to mathematics, and drew the conclusion that the U.S.S.R. is unequalled in its ability to apply mathematical theories to practical problems. They commented on the large number of Soviet engineers who keep up with the latest mathematical developments and exploit the deepest kind of mathematics. If time spent on learning something of the rather more abstract developments of modern mathematics will mean a significant economy of time and effort at the stage of practical application, can we give the young technologist not only the time but also the taste and incentive to make such a study? T. A. A. BroAdBent

\section{ELEMENTARY NUCLEAR PHYSICS}

Nuclear Physics

By Irving Kaplan. (Addison-Wesley Series in Nuclear Science and Engineering.) Pp. xiv +770 . (Reading, Massachusetts, Palo Alto and London: Addison-Wesley Publishing Company, Inc., 1963, second edition.) $64 s$.

THERE seems to be a widespread predilection among the writers of physics books to overestimate the capacities and comprehension of the readers to whom their books are addressed. Thus one finds that many undergraduate texts are appreciated and studied in detail only at graduate level while the presentation of graduate texts often taxes the understanding of the experienced professional physicist, and theoretical treatments aimed at the experimentalist all too frequently fall short of their mark. One result of this must surely be the generation of diffidence and despondency in the average reader of such 